Use of compound specific 13C stable isotope analysis for intact triacylglcerides using high temperature gas chromatography up to $430 \mathrm{C}$.

\title{
Sutton, $\mathrm{P}$
}

http://hdl.handle.net/10026.1/15056

10.26434/chemrxiv.9933236.v1

ChemRxiv

All content in PEARL is protected by copyright law. Author manuscripts are made available in accordance with publisher policies. Please cite only the published version using the details provided on the item record or document. In the absence of an open licence (e.g. Creative Commons), permissions for further reuse of content should be sought from the publisher or author. 


\section{Use of compound specific $\delta^{13} \mathrm{C}$ stable isotope analysis for intact triacylglcerides using high temperature gas chromatography up to $430^{\circ} \mathrm{C}$.}

Paul Antony Sutton ${ }^{*}$, Mario Tuthorn ${ }^{\mathrm{b}}$ and Jason Newton ${ }^{\mathrm{c}}$

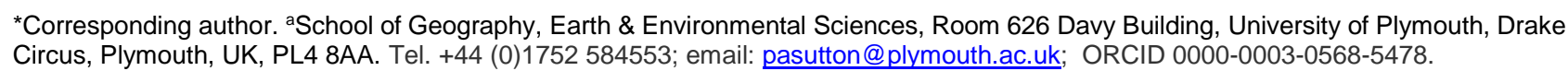
${ }^{\text {b} T h e r m o ~ F i s h e r ~ S c i e n t i f i c, ~ H a n n a-K u n a t h-S t r . ~ 11, ~} 28199$ Bremen, Germany. Tel. +49 (0) 4215493 335; email: Mario.tuthorn@thermofisher.com

'Isotope Community Support Facility, Scottish Universities Environmental Research Centre (SUERC), Rankine Avenue, East Kilbride, Glasgow, G75 0QF. Tel. +44 (0)1355 270138; email: Jason.Newton@glasgow.ac.uk; ORCID 0000-0001-7594-3693.

\section{Abstract}

We report a preliminary study demonstrating that high temperature gas chromatography with the oven operated at up to $430^{\circ} \mathrm{C}$ can be coupled with compound specific isotope ratio mass spectrometry (HTGC-CSIRMS) for the measurement of stable carbon isotopes. There was good agreement between data obtained using bulk and compound specific techniques for the $\mathrm{C}_{87}$ hydrocarbon apolane-87 and tripalmitolein. $\delta^{13} \mathrm{C}$ values of three main triacylglycerides in different olive oils were measured for the first time and differed from bulk values of the olive oils. The use of HTGC-CSIRMS has wide-ranging application and warrants further study and validation.

Keywords

High temperature gas chromatography $\delta^{13} \mathrm{C}$ compound specific isotope ratio mass spectrometry.

Chemical compounds studied in this article

Tripalmitolein (Pubchem CID: 9543989); 24,24-deithyl-19,29-dioctadecylheptatetracontane (Pubchem CID: 12546230).

\section{Introduction}

Esters of glycerol and three fatty acids (triacylglycerides or TAGs; Figure 1) are ubiquitous components of vegetable oils and animal fats and are incorporated in many foodstuffs. TAG distributions can be complex owing to differences in the structure of the fatty acids (FAs) and their site of attachment to the glycerol (sn-1, sn-2 or sn3). Methyl transesterification is the most usual procedure for liberating the individual free fatty acids (FFAs) from the TAG mixture as their fatty acid methyl esters (FAMEs) in order to undertake routine analysis for quality, source and authenticity assurance (European Commission 1991). Gas chromatography (GC) where the oven is cycled up to around $300^{\circ} \mathrm{C}$ enables efficient separation of individual FAMEs using a suitable chromatographic column. When coupled with flame ionisation detection (FID) this approach can be used for quantitative analysis where response factors for individual FAMEs are approximately equivalent. Coupling of GC with mass spectrometry (GC-MS) allows the molecular characterisation of the separated FAMEs using their 'fingerprint' mass spectra and relative retention times (Farkas et al., 2008). Liquid chromatographic (LC) techniques with 
ultraviolet detection and MS have also been used to identify and quantify FAMEs (e.g. Wei and Zeng 2011). Similarly, LC coupled with refractive index detection or MS (electrospray ionisation, atmospheric pressure chemical ionisation, atmospheric pressure photoionisation or matrix assisted laser desorption ionisation; ESI, APCI, APPI or MALDI) have been used for intact TAGS, as recently reviewed by Tena et al 2019. In addition to quantitative analysis and molecular identification, measurement of the stable isotopes of constituent carbon, hydrogen and oxygen atoms can provide a profile of the biological, chemical and physical processes that each molecule has undergone (Lichtfouse 2000). Stable isotopic values of individual FAMEs (compound specific) are usually obtained when GC is coupled with a stable isotope ratio mass spectrometer (GC-IRMS; Camin et al., 2010; Paolini et al.,

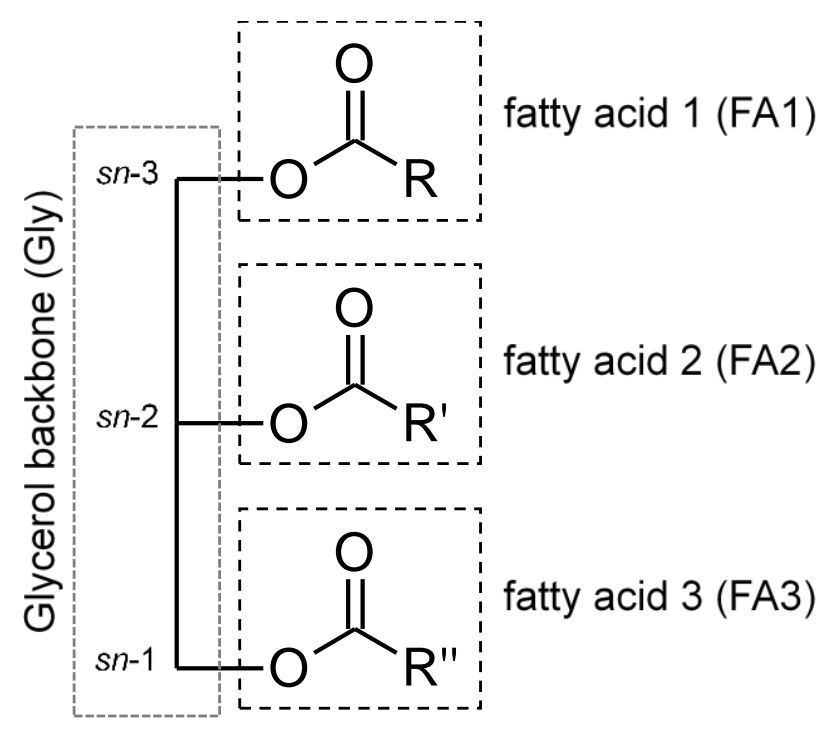

2017). Methyl esterification has been reported to have a modest effect on the $\delta^{13} \mathrm{C}$ and $\delta^{2} \mathrm{H}$ values of FAMEs (Chivall et al., 2012; Paolini et al. (2017). Although LC has been coupled with IRMS it is less well established due to difficulties removing organic solvent necessary to effect some chromatographic separations. When GC is coupled to the IRMS via a catalytic combustion reactor operated at around $1000^{\circ} \mathrm{C}$ (GC-C-IRMS) the FAMEs are converted to $\mathrm{CO}_{2}$ and the $\delta^{13} \mathrm{C}$ of the $\mathrm{CO}_{2}$ measured relative to a reference gas. Similarly, $\delta^{2} \mathrm{H}$ (including $\mathrm{H}_{3}{ }^{+}$correction) and $\delta^{18} \mathrm{O}$ can be measured from $\mathrm{H}_{2}$ and $\mathrm{CO}$, respectively, when the $\mathrm{GC}$ is interfaced with the IRMS via a pyrolysis reactor (GC-Py-IRMS; preconditioned with solvent) and operated at around $1420^{\circ} \mathrm{C}$. Only recently has the GC-Py-IRMS $\delta^{2} \mathrm{H}$ measurement of FAMEs been reported (Paolini et al., 2017).

Figure 1 Schematic representation of triacylglyceride components $\left(R, R^{\prime}\right.$ and R" represent substituent saturated, unsaturated, phenolic or hydroxylated hydrocarbon chains, and as the corresponding fatty acids: FA1, FA2 and FA3 may have the same or different structures).

Carbon fractional isotopic abundance $\left({ }^{13} \mathrm{~F}\right.$; Eq 1), carbon isotope ratio $\left({ }^{13} \mathrm{R} ; \mathrm{Eq} 2\right)$ and the representation of isotope values using the delta notation $\left(\delta^{13} \mathrm{C}\right)$ can be calculated from isotope measurements ${ }^{13} F=\frac{{ }^{13} R}{1+{ }^{13} R}$ Eq 1.

${ }^{13} R=\frac{{ }^{13} C}{{ }^{12} C}$ Eq 2.

$\delta^{13} C=\left[\left(\frac{{ }^{13} R_{\text {standard }}}{{ }^{13} R_{\text {sample }}}\right)-1\right] \times 1000$ Eq 3.

$F F A \delta^{13} C=\frac{\left(F A M E_{C} \times F A M E \delta^{13} C\right)-m e t h y l \delta^{13} C}{F F A_{C}} \quad$ Eq 4 Where $F A M E_{C}$ is the number of carbon atoms in the $F A M E$, FAME $\delta^{13} \mathrm{C}$ is the measured $\delta^{13} \mathrm{C}$, methyl $\delta^{13} \mathrm{C}$ is methyl $\delta^{13} \mathrm{C}$ derived from comparative isotopic analysis of FFAs and FAMEs, and FFA is the number of carbon atoms in the free fatty acid (Morrison et al., 2010).
(Eq 3). An adjustment is made for the isotopic contribution of the chemical derivatisation (usually methyl-) group (Eq 4; Morrison et al., 2010).
Following from the above, the mass balance equation between a TAG and its constituents can be written as Equation 5:

$n C_{T A G} F_{T A G}=n C_{G l y} F_{G l y}+n C_{F A 1} F_{F A 1}+n C_{F A 2} F_{F A 2}+n C_{F A 3} F_{F A 3}$ Eq 5. 
Where $\mathrm{nC}$ represents the molar quantity of carbon in the parent TAG and constituent glycerol backbone and fatty acid moieties and $F$ is the fractional isotopic abundance (Eq 1). Bulk isotope analysis of an individual TAG can be used to represent the left hand side of Eq 5 (where $\mathrm{F}_{\mathrm{TAG}}=1$ ) and CSIRMS used to determine the (derivative adjusted) value for FA1, FA2 and FA3. In this way the isotopic contribution due to the glycerol moiety can be estimated by solving the equation. Alternatively the isotopic value of glycerol can be measured directly when recovered from the saponification liquor (Fronza et al., 1998). However, where more than one TAG is present the isotopic value measured for the bulk TAGs then represents an average value. Cleavage of glycerol during saponification or transesterification of FAMEs from TAGs means that the intact structure and isotopic value of the TAG is lost, and the distribution and isotopic composition of cleaved FAs and glycerol are averaged. In complex TAG mixtures the parent TAG isotopic data cannot be calculated using this approach and potentially useful isotopic information of individual TAGs is lost. For natural samples such as olive oil, bulk isotope measurements are dominated by the contribution from TAGs but also reflect the presence of other organic compounds e.g. FFAs, sterols, glycerol, pigments and flavour compounds (Fronza et al., 1998; Eq 6).

$n C_{O O} F_{O O}=n C_{T A G S} F_{T A G S}+n C_{F F A S} F_{F F A s}+n C_{\text {Sterols }} F_{\text {Sterols }}+n C_{G l y} F_{G l y}+n C_{\text {Pig }} F_{\text {Pig }}+n C_{\text {Flav }} F_{F l a v} \quad$ Eq 6.

Where OO represents the bulk olive oil $\delta^{13} \mathrm{C}$ value $\left(\mathrm{F}_{\mathrm{OO}}=1 ; \sim 99 \%\right.$ of which is due to TAGs Boskou et al. 2006) and other terms represent the contributions (molar carbon $\mathrm{nC}$ and fractional isotopic abundance F) from free fatty acids (FFAs), sterols, glycerol (Gly), pigments (Pig) and flavour compounds (Flav).

The catalytic thermal conversion of organic compounds occurs at a higher temperature than that used to separate compounds on the gas chromatographic (GC) column (typically $\sim 330-350^{\circ} \mathrm{C}$, above which is generally described as high temperature gas chromatography; Blum and Aicholz 1991). Therefore, the temperature limit employed in the GC oven is not the limiting factor to achieve CSIRMS. GC oven temperatures up $420^{\circ} \mathrm{C}$ have been used for analysis of intact TAGs with FID (Mayer and Lorbeer 1997; Buchgraber et al., 2004 and references therein) and MS (Ruiz-Samblás et al., 2010; Sutton and Rowland 2012) and TAG analysis using HTGC has been recently reviewed for a wide range of foodstuffs (Ruiz-Samblás et al., 2015). Flow splitting to allow dual detection (FID and MS) for qualitative and quantitative analysis of FAs, mono-, di- and triacylglycerols in biodiesel has been reported with HTGC up to $380^{\circ} \mathrm{C}$ (Flanagan et al., 2019). HTGC has also been used for other high boiling components of food e.g. oligosaccharides (Carlsson et al., 1992), glycosides (Isidorov and Nazaruk 2017), wax esters (Pereira et al., 2002), triterpenes (Pereira et al., 2003), and in authentication studies e.g. beeswax (Maia and Nunes 2013). In this sense it is surprising that there is a paucity of literature detailing the coupling of HTGC with combustion and/or pyrolysis IRMS for compound specific analysis. We could find only one reference detailing the coupling of HTGC operated up to $420^{\circ} \mathrm{C}$ with IRMS. Carlson et al. (1997) presented data suggesting little difference in the carbon isotope values of lower molecular weight $n$-alkanes $\left(\mathrm{C}_{20-40}\right)$ compared to higher molecular weight $n$-alkanes and iso- and/or monocyclic analogues $\left(\mathrm{C}_{57-65}\right)$ in samples of solid bitumen. It is unclear why the methodology was not pursued further. Some twenty years later, particular technological advances such as GC electronic flow control, robust air-tight connectors (micro fluidic devices and steel or silver ferrules) and capillary reactors suggest that this approach may regain some momentum. We hypothesised that these developments would enable us to undertake carbon CSIRMS analysis with compounds eluting from a GC column at up to $430^{\circ} \mathrm{C}$. Here we present preliminary data comparing $\delta^{13} \mathrm{C}$ isotope data obtained using bulk and compound specific isotope ratio mass spectrometry with the latter utilising HTGC operated up to $430^{\circ} \mathrm{C}$.

\section{Materials and methods}

\subsection{Chemicals and samples}

Tripalmitolein ( $\mathrm{I} ; \geq 98 \%$ ) was purchased from Sigma-Aldrich, UK. 24,24-diethyl-19,29-dioctadecylheptatetracontane (II; apolane-87) was a legacy sample previously gifted to our laboratory. Commercially available olive oils used in this work were: OIL1, extra virgin olive oil (purchased in Cyprus) and OIL2, olive oil composed of virgin and refined (Spanish) olive oils (Colic 2019, Pers. Commun.).

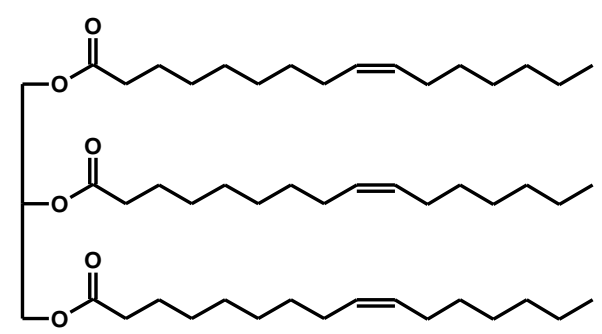

Structure I tripalmitolein

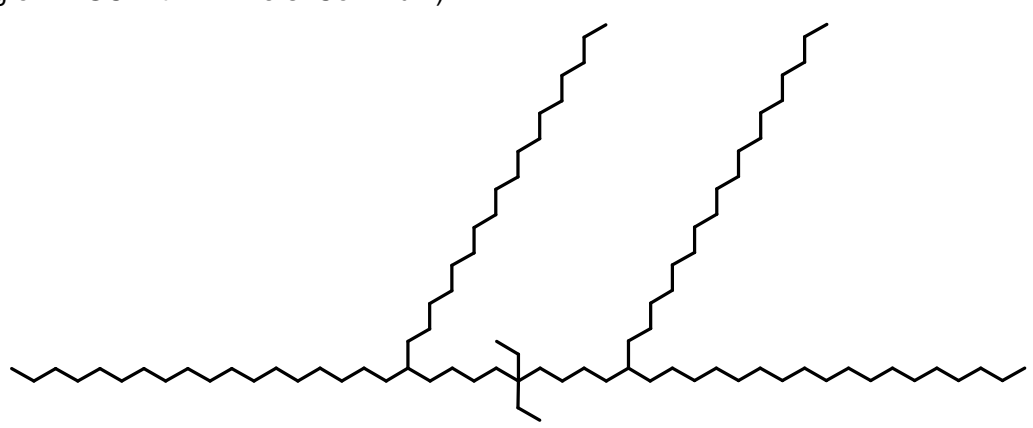

Structure II 24,24-diethyl-19,29-dioctadecylheptatetracontane (apolane-87) 


\subsection{Methyl trans-esterification of tripalmitolein}

An aliquot of tripalmitolein was partially methyl trans-esterified by heating in a closed vial with $10 \% \mathrm{HCl} / \mathrm{MeOH}\left(\mathrm{v} / \mathrm{v} ; 2 \mathrm{~mL} ; 70^{\circ} \mathrm{C} ; 2\right.$ h). The extract was recovered, following the addition of water (2 $\mathrm{mL})$, in cyclohexane ( $3 \times 2 \mathrm{~mL})$ after mixing and centrifugation. Solvent was removed $\left(\mathrm{N}_{2} / 65^{\circ} \mathrm{C}\right)$ and the extract re-dissolved in cyclohexane. The incomplete methyl trans-esterification of tripalmitolein allowed the CSIRMS carbon isotope values of the intact TAG and FAME derivative to be measured in the same analysis.

\subsection{High temperature gas chromatography with flame ionisation detection (HTGC-FID)}

Sample was injected $(0.5 \mu \mathrm{L})$ via a cool-on-column inlet (track oven mode; $+3^{\circ} \mathrm{C}$ ) onto a MXT-biodiesel TG column (15 m x 0.32 $\mathrm{mm} \times 0.10 \mu \mathrm{m}$; Thames Restek Ltd., UK) operated with helium carrier gas (constant flow mode; $1 \mathrm{~mL} \mathrm{~min}^{-1}$ ) and the $\mathrm{GC}$ oven (HP6890) programmed from $40-370^{\circ} \mathrm{C}$ at $10^{\circ} \mathrm{C} \mathrm{min}^{-1}$ with 10 minute hold. The FID was operated at $375^{\circ} \mathrm{C}$ (FID gas flows optimised at $\mathrm{H}_{2} 40 \mathrm{~mL} \mathrm{~min}^{-1}$, air $450 \mathrm{~mL} \mathrm{~min}^{-1}$ and $\mathrm{N}_{2}$ make-up 45 $\mathrm{mL} \mathrm{min}^{-1}$ ).

2.4 High temperature gas chromatography with time-of-flight mass spectrometry (HTGC-ToF-MS)

Sample was injected $(0.5 \mu \mathrm{L})$ via a cool-on-column inlet (track oven mode; $\left.+3^{\circ} \mathrm{C}\right)$ onto a Vf-5ht Ultimetal column $(15 \mathrm{~m} \times 0.25 \mathrm{~mm}$ x $0.10 \mu \mathrm{m}$; Agilent Technologies Ltd., UK) operated with helium carrier gas (constant flow mode; $2 \mathrm{~mL} \mathrm{~min}^{-1}$ ) and the GC (HP6890; Agilent Technologies, UK) oven programmed from $40-430^{\circ} \mathrm{C}$ at $20^{\circ} \mathrm{C} \mathrm{m^{-1 }}$ with a 2 minute isothermal hold. The column was connected with a Siltite ${ }^{\mathrm{TM}}$ mini union via a high temperature silica transfer capillary (nom. $2 \mathrm{~m} \times 0.18 \mathrm{~mm}$ ) through a vendor modified heated $\left(380^{\circ} \mathrm{C}\right)$ transfer line into a BenchToF Select eV (Markes International Ltd, UK) with the ion source at $230^{\circ} \mathrm{C}$. ToF-DS software (Markes International Ltd, UK) was used to operate the mass spectrometer (tuned at $12 \mathrm{eV}$ ) and record ( $\mathrm{m} / \mathrm{z} 50-950)$ and process data.

$2.5{ }^{13} \mathrm{C}$ High temperature gas chromatography compound specific combustion isotope ratio mass spectrometry (HTGC-CCSIRMS)

Compound specific carbon isotope ratio mass spectrometry analysis was undertaken using a Thermo Scientific ${ }^{\mathrm{TM}}$ IRMS instrument: a TRACE ${ }^{\mathrm{TM}} 1310 \mathrm{GC}$ (schematic Figure 2) connected to a GC IsoLink II ${ }^{\mathrm{TM}}$ conversion unit, interfaced to a DELTA $V^{\mathrm{TM}}$ isotope ratio mass spectrometer via a ConFlo IV ${ }^{\mathrm{TM}}$ Universal Interface. A $1 \mu \mathrm{L}$ aliquot was manually injected on-column through a Merlin Microseal ${ }^{\mathrm{TM}}$ high pressure valve onto a Vf-5ht Ultimetal column (30 m x 0.25 mm x $0.10 \mu \mathrm{m}$; Agilent Technologies UK Ltd., UK) with a retention gap ( $2 \mathrm{~m} \times 0.53 \mathrm{~mm}$; Agilent Technologies UK Ltd., UK) and the GC oven programmed at $20^{\circ} \mathrm{C} \mathrm{min}^{-1}$ from $50^{\circ} \mathrm{C}$ to final temperatures of $390-430^{\circ} \mathrm{C}$ and final isothermal time from 5-15 minutes, with helium carrier gas (1.4 or $\left.1.5 \mathrm{~mL} \mathrm{~min}^{-1}\right)$. The chromatographic column was coupled to dual capillary ceramic reactors (combustion reactor comprised $\mathrm{NiO}$ tube with $\mathrm{CuO}, \mathrm{NiO}$ and $\mathrm{Pt}$ wires at $1000^{\circ} \mathrm{C}$; high temperature pyrolysis reactor at $1420^{\circ} \mathrm{C}$ but not used in the current work) via a micro channel device (MCD). Deactivated steel coated silica capillary tubing was soldered to the capillary reactor inlet ends and MCD connections (including helium flow connection) were made using silver ferrules. Isodat $^{\mathrm{TM}}$ software suite (Thermo Scientific ${ }^{\mathrm{TM}}$ ) was used for data acquisition and evaluation. 


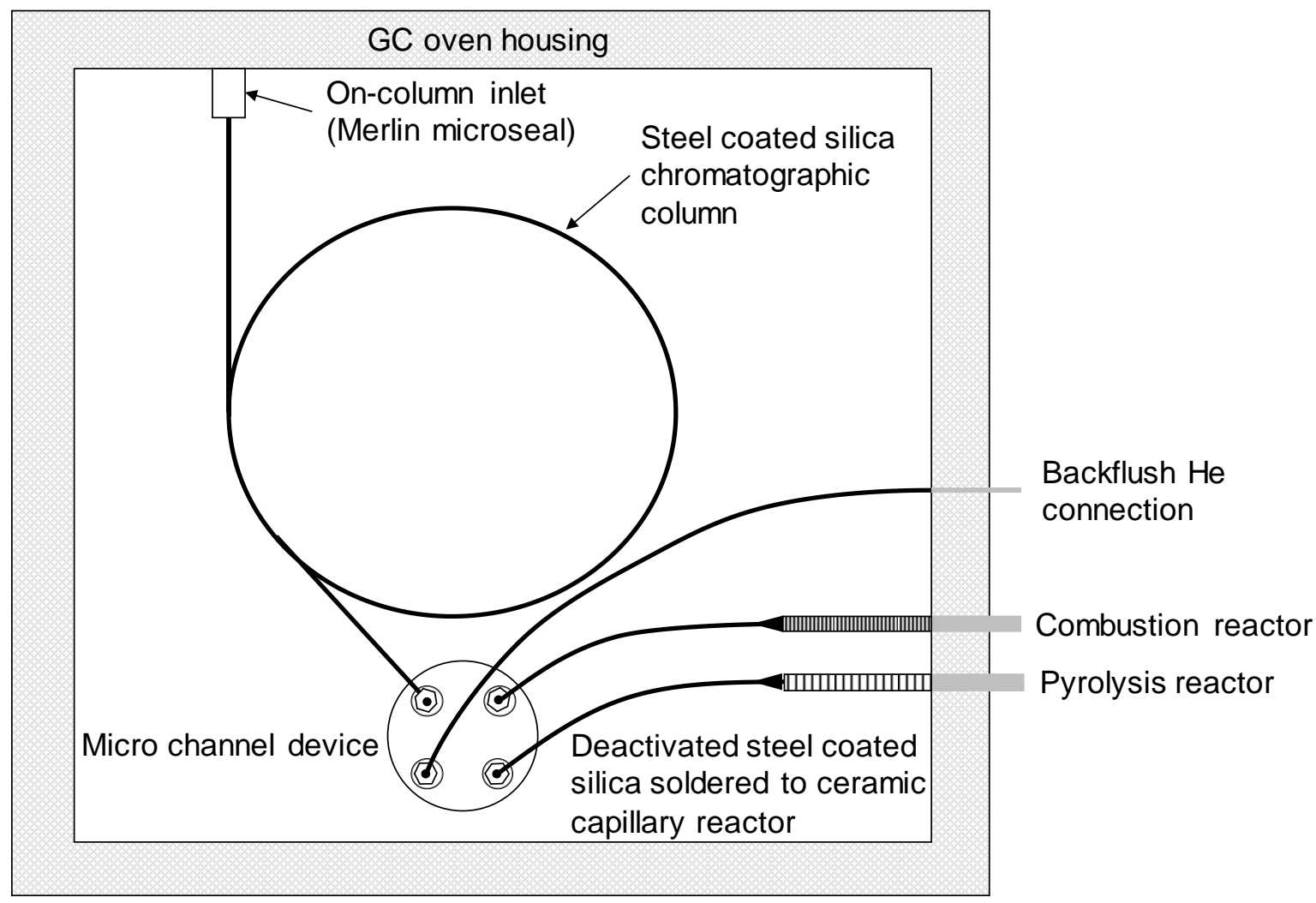

Figure 2 Schematic of Thermo Scientific ${ }^{\mathrm{TM}}$ TRACE $^{\mathrm{TM}} 1310 \mathrm{GC}$ set-up for high temperature gas chromatography-combustion-compound specific isotope ratio mass spectrometry.

\subsection{Bulk carbon isotope analysis}

$25 \mu \mathrm{L}$ sample aliquots (10 $\mathrm{mg} \mathrm{mL}^{-1} ; 0.25 \mathrm{mg}$ sample) were dispensed into silver capsules and solvent removed by evaporation (heater plate, $50^{\circ} \mathrm{C}$ ) prior to folding and loading into the autosampler of an Elementar Pyrocube elemental analyser, which converted the $\mathrm{C}$ in the samples to $\mathrm{CO}_{2}$ which was measured for $\delta^{13} \mathrm{C}$ on a Thermo Fisher Scientific Delta XP Plus IRMS. Inhouse reference materials: GEL (gelatin solution, $\delta^{13} \mathrm{C}=-20.02 \%$ ), ALAGEL (alanine-gelatine solution spiked with ${ }^{13} \mathrm{C}$-alanine, 9.23\%o), and GLYGEL (glycine-gelatine solution, $-38.58 \%$ ), each dried for 2 hours at $70^{\circ} \mathrm{C}$; and four USGS 40 glutamic acid standards (Qi et al., 2003; Coplen et al., 2006) were used as independent checks of accuracy.

\section{Results and Discussion}

A cross-plot (Figure 3 ) of replicate bulk and compound specific isotope ratio measurements (Table 1) shows good agreement between $\delta^{13} \mathrm{C}$ values for two synthetic standards, tripalmitolein and apolane-87, even where apolane- 87 was eluted at $430^{\circ} \mathrm{C} \mathrm{GC}$ oven temperature. The mean compound specific $\delta^{13} \mathrm{C}$ value of intact tripalmitolein (-27.11 \%o $\pm 0.08 \%$; $n=3$; Table 1) was not significantly different (student's t-test, $p=0.18$ ) from the bulk $\delta^{13} \mathrm{C}$ isotope value obtained for the neat TAG $(-27.31 \% \pm 0.20 \%$; $n=$ 3; Table 1). Bulk tripalmitolein measurements were obtained using $0.25 \mathrm{mg}$ samples ( $191 \mu \mathrm{g} \mathrm{C}$; $\mathrm{C}=76.45 \%$ of $\mathrm{TAG}$ ) whereas the CSIRMS measurements were obtained with either approximately 10 or $20 \mathrm{ng}$ of TAG ( 7.6 or $15.3 \mathrm{ng} \mathrm{C}$ ) injected on column and tripalmitolein eluting at $390^{\circ} \mathrm{C}$ (e.g. Figure S1). Similarly, the mean CSIRMS $\delta^{13} \mathrm{C}$ isotope value $(-32.39 \% \pm 0.42 \% ; n=4$; Table $1 ; 43$ ng $\mathrm{C}$ on column) of apolane-87, which eluted at $430^{\circ} \mathrm{C}$ (e.g. Figure $\mathrm{S} 2$ ), was not statistically different (student's t-test, $p=0.43$ ) from the bulk $\delta^{13} \mathrm{C}$ isotope value obtained for apolane-87 (-32.61\%o $\pm 0.07 \% ; n=3 ; 214 \mu \mathrm{g} \mathrm{C}$; Table 1). 


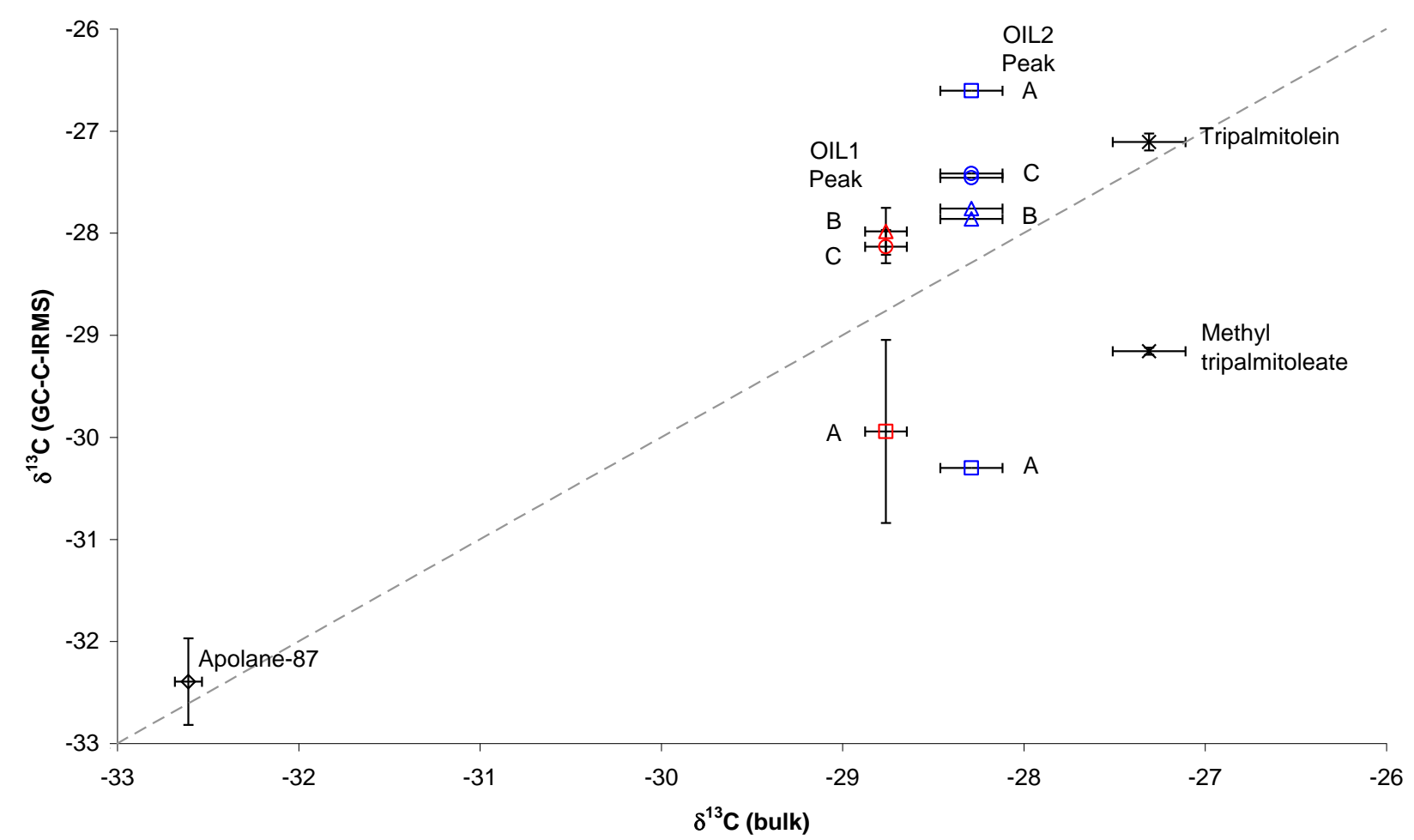

Figure 3 Crossplot of $\delta^{13} \mathrm{C}$ obtained using EA-C-IRMS (x-abscissa) and $\delta^{13} \mathrm{C}$ obtained using GC-C-IRMS (y-abscissa). Measurements are in \% notation relative to Pee Dee Belemnite (PDBE; $n=3$ for apolane-87, tripalmitolein, methyl tripalmitoleate and OIL1; $n=2$ for OIL2; error bars represent $1 \mathrm{SD})$.

Table 1 Bulk and high temperature gas chromatography compound specific isotope ratio $\delta^{13} \mathrm{C}$ values for samples analysed in this study.

\begin{tabular}{|l|c|c|}
\hline \multicolumn{1}{|c|}{ Sample } & Bulk $\delta^{13} \mathrm{C}$ & HTGC-C-IRMS $\delta^{13} \mathrm{C}$ \\
\hline Apolane-87 & $-32.58,-32.69,-32.55$ & $-31.88,-32.21,-32.75,-32.73$ \\
\hline \multirow{2}{*}{ Tripalmitolein } & $-27.32,-27.51,-27.11$ & $-27.04,-27.08,-27.20$ \\
\hline \multirow{3}{*}{ Methyl tripalmitoleate } & \multirow{3}{*}{ Not measured } & -29.20 \\
\cline { 2 - 3 } & & -29.13 \\
\hline \multirow{3}{*}{ OIL1 } & -28.83 & -29.14 \\
\cline { 2 - 3 } & -28.83 & $-28.99^{\mathrm{a}},-30.08^{\mathrm{a}},-30.77^{\mathrm{a}}$ \\
\hline \multirow{3}{*}{ OIL2 } & -28.63 & $-27.21^{\mathrm{b}},-27.76^{\mathrm{b}},-27.98^{\mathrm{b}},-28.12^{\mathrm{c}},-28.30^{\mathrm{c}}$ \\
\cline { 2 - 3 } & -28.34 & $-30.30^{\mathrm{a}},-26.60^{\mathrm{a}}$ \\
\cline { 2 - 3 } & -28.43 & $-27.86^{\mathrm{b}},-27.76^{\mathrm{b}}$ \\
\cline { 2 - 3 } & -28.10 & $-27.42^{\mathrm{c}},-27.46^{\mathrm{c}}$ \\
\cline { 2 - 3 } & &
\end{tabular}

${ }^{a}$ Peak A, ${ }^{b}$ peak B and ${ }^{c}$ peak C in Figure S6 and S10 for OIL1 and OIL2, respectively.

Partial methyl trans-esterification of tripalmitolein enabled us to obtain compound specific $\delta^{13} \mathrm{C}$ values for the intact triacylglyceride $(-27.11 \% \pm 0.08 \% ; n=3)$ and methyl palmitoleate (-29.16\% $\pm 0.03 \% ; n=3$ ) in the same chromatographic analysis (Figure $S 1$; S3 and S4) in order to investigate the difference between measuring TAGs and the more routinely reported FAMEs. $\delta^{13} \mathrm{C}$ values of tripalmitolein and methyl palmitoleate were significantly different (student's t-test, $p=3 e^{-6}$ ) with the mean value of the recovered FAME around $2 \%$ heavier than the parent TAG. This difference is either related to the $\delta^{13} \mathrm{C}$ contribution of the cleaved glycerol (source unknown but values are typically lighter than -20 $\%$; Fronza et al 1998) or the addition of the esterifying methyl group, or both. Either way the calculated $\delta^{13} \mathrm{C}$ of glycerol would be $5.70 \%$ (Supplementary Material) so it is difficult to reconcile a 2 $\%$ shift with the loss and/or gain of three carbon atoms and additional studies are warranted. 
High temperature gas chromatography can be used to characterise the distribution of TAGs in different vegetable oils (Figure S5). However, the heterogeneity of TAGs between different olive oil brands is less obvious (Figure S6) and isotope ratio techniques could be usefully applied. Using bulk analysis of olive oil samples OIL2 (-28.29\%o $\pm 0.17 \%, n=3$; Figure 3 ) and Oil $8(-28.76 \% \pm 0.12 \% ; n=3$; Figure 3$)$ it was possible to statistically differentiate these samples based on their ${ }^{13} \mathrm{C}$ isotope ratios. Carbon isotope ratios of olive oils are influenced by a complex of factors such as latitude, water availability, coastal proximity, temperature and relative humidity (Camin et al., 2010; Chiocchini et al., 2016). We were interested to see whether this difference was evident in dominant constituent TAGs.

The present study used a Vf-5ht column to separate the main TAGs prior to combustion for isotope analysis which may have led to some overlap with minor components (e.g. Figure S4 where OIL2 and Oil 8 were separated on an MXT- biodiesel TG column). Nevertheless, peaks A-C for OIL2 and Oil 8 were identified using HTGC-ToF-MS (Supplementary Material) as TAGs principally containing one oleic and two palmitic acids $(A)$, one palmitic and two oleic acids (B) and three oleic acids (C), consistent with previous work where HTGC was used to analyse olive oil TAGs (Ruiz-Samblás et al., 2012). HTGC carbon CSIRMS of the three major peaks (peaks $\mathrm{A}, \mathrm{B}$ and $\mathrm{C}$ ) in the chromatogram of Oil 8 (Figure S5) produced $\delta^{13} \mathrm{C}$ values that were not significantly different to the bulk $\delta^{13} \mathrm{C}$ value $(-28.76 \% \pm 0.12 \% ; n=3)$ for peak A $(-29.94 \% \pm 0.90 \% ; n=3$; Student's t-test, $p=0.086)$ but heavier than the bulk value for peaks $B(-27.98 \% \pm 0.23 \% ; n=3$; student's t-test, $p=0.006)$ and $C(-28.13 \% 0 \pm 0.16 \% ; n=3$; student's t-test, $p=0.005)$. The values for peaks $B$ and $C$ were around 0.78 and $0.63 \%$ heavier than the bulk value, respectively, but not statistically different from each other (student's $t$-test, $p=0.41$ ). One of the replicate $50 \mathrm{ng}$ on column injections produced peaks that were around four times higher than the other two replicates, gave lighter values for all three peaks and was most variable for peak $A$, which may relate to linearity. OIL2 was analysed twice with injections of 50 and $100 \mathrm{ng}$ on column. Whilst there was an order of magnitude difference in peak amplitude the $\delta^{13} \mathrm{C}$ values for peaks $B$ and $C$ were reasonably consistent whilst the smallest peak $A$ varied by around $3.6 \%$ (Figure 3 ).

\section{Conclusions}

This preliminary work has confirmed our hypothesis and demonstrated for the first time that high temperature gas chromatography using GC oven temperatures up to $430^{\circ} \mathrm{C}$ can be coupled with compound specific isotope ratio mass spectrometry for the measurement of carbon isotopes. Measurement of $\delta^{13} \mathrm{C}$ values of discrete compounds using bulk and CSIRMS analytical approaches indicated that results were consistent when employing high temperature $\mathrm{GC}$ separation. Online $\delta^{13} \mathrm{C}$ values of intact triglycerides in olive oil have been obtained for the first time following HTGC separation. The direct measurement of parent compound stable isotopes reduces sample preparation time dispensing with the need for sample work-up and derivatisation procedures. This approach has potential uses in studies of food authenticity, adulteration and quality control, industrial feedstock chemicals and products, petroleum, and microbial lipid (e.g. glycerol dialkyl glycerol tetraethers) analysis. Future studies should include more extensive sample analysis to more rigorously optimise instrumental settings and method validation including the importance of injection volume and more extensive repeatability. The development of new column phases capable of withstanding high temperature operation (e.g. ionic liquids) may help overcome some of the separation limitations due to the current restricted choice of column phases. Whilst this preliminary study has investigated stable carbon isotope analysis, future studies may investigate its application to hydrogen and oxygen isotope measurements.

\section{Acknowledgements}

This research did not receive any specific grant from funding agencies in the public, commercial, or not-for-profit sectors.

\section{References}

Boskou, D., Blekus, G. And Tsimidou, M. Olive Oil Composition. In, (Ed. Boshou, D.) Olive Oil. Academic Press and AOCS Press. Pp 282.

Blum, W. and Aicholz, R. (1991). Hochtemperatur gaschromatographie, Hüthig Verlag, Heidelberg. Cited in Aicholz, R. and Lorbeer, E. (1998). Separation of triacylglycerolds by high temperature gas chromatography on seven different stationary phases. Journal of High Resolution Chromatography, 21, 363-367.

Buchgraber, M., Ulbeth, F., Emons, H. and Anklam, E. (2004). Triacylglycerol profiling by using chromatographic techniques. European Journal of Lipid Science and Technology, 106, 621-648.

Camin, F., Larcher, R., Perini, M., Bontempo, L., Bertoldi, D., Gagliano, G., Nicolini, G. and Versini, G. (2010). Characterisation of authentic Italian extra-virgin olive oils by stable isotope ratios of $\mathrm{C}, \mathrm{O}$ and $\mathrm{H}$ and mineral composition. Food Chemistry, 118, 901909.

Carlson, R.M.K., Dias, R.F. and Schoell, M. Origins of high molecular weight alkanes $>\mathrm{C} 40$ in waxes from natural crude oils and bitumens based on carbon isotopic evidence. 28th International Meeting on Organic Geochemistry, 22-26 September 1997, Maastricht, The Netherlands. Forschungszentrum Jülich $\mathrm{GmbH}$, Germany. 
Carlsson, N-G., Karlsson, H. and Sandberg, A-S. (1992). Determination of oligosaccharides in foods, diets, and intestinal contents by high-temperature gas chromatography and gas chromatography/mass spectrometry. Journal of Agriculture and Food Chemistry, 40, 2404-2412.

Chang, T.L. and Li, W-J. (1990). A calibrated measurement of the atomic weight of carbon. Chinese Science Bulletin, 35, 290-296.

Chiocchini, F., Portarena, S., Ciolfi, M., Brugnoli, E. and Lauteri, M. (2016). Isoscapes of carbon and oxygen stable isotope compositions in tracing authenticity and geographical origin of Italian extra-virgin olive oils. Food Chemistry, 202, 291-301.

Chivall, D., Berstan, R., Bull, I.D. and Evershed, R.P. (2012). Isotope effects associated with the preparation and methylation of fatty acids by boron trifluoride in methanol for compound-specific stable hydrogen isotope analysis via gas chromatography/thermal conversion/isotope ratio mass spectrometry. Rapid Communications in Mass Spectrometry, 26, 1232-1240.

Colic, I. Thornton \& Ross Limited, UK. (2019) Pers. Commun.

Coplen, T.B., Brand, W.A., Gehre, M., Gröning, M., Meijer, H.A.J., Toman, B. and Verkouteren, R.M. (2006). New guidelines for $\delta^{13} \mathrm{C}$ measurements. Analytical Chemistry, 78, 2439-2441.

Coplen, T.B. and Qi, H. (2010). Applying the silver-tube introduction method for thermal conversion elemental analyses and a new $\delta^{2} \mathrm{H}$ value for NBS 22 oil. Rapid Communications in Mass Spectrometry, 24, 2269-2276.

European Commission (1991) European Commission Regulation (EEC) No 2568/91.

Farkas, O., Zenkevich, I.G., Stout, F., Kalivas, J.H. and Héberger, K. (2008). Prediction of retention indices for identification of fatty acid methyl esters. Journal of Chromatography A, 1198-1199, 188195.

Flanagan, G., Andrianova, A.A., Casey, J., Hellrung, E., Diep, A.B., Seames, W.S. and Kubátová, A. (2019). Simultaneous hightemperature gas chromatography with flame ionization and mass spectrometric analysis of monocarboxylic acids and acylglycerols in biofuels and biofuel intermediate products. Journal of Chromatography A, 1584, 165-178.

Fronza, G., Fuganti, C., Grasselli, P., Reniero, F., Guillou, C., Breas, O., Sada, E., Rossmann, A. and Hermann, A. (1998). Determination of the ${ }^{13} \mathrm{C}$ Content of glycerol samples of different origin. Journal of Agriculture and Food Chemistry, 46, 477-480.
Gerstenberger, H., and Herrmann, M. (1983). Report on the intercomparison for the isotope standards limestone $\mathrm{KH} 2$ and polyethylene foil PEF 1 (Zfl-Mitt--66). German Democratic Republic.

Isidorov, V.A. and Nazaruk, J. (2017). Gas chromatographic-mass spectrometric determination of glycosides without prior hydrolysis. Journal of Chromatography A, 1521, 161-166.

Lichtfouse, E. (2000). Compound-specific isotope analysis (CSIA). Application to archaeology, biomedical sciences, biosynthesis, environment, extraterrestrial chemistry, food science, forensic science, humic substances, microbiology, organic geochemistry, soil science and sport. Rapid Communications in Mass Spectrometry, 14, 1337-1344.

Maia, M. and Nunes, F.M. (2013). Authentication of beeswax (Apis mellifera) by high- temperature gas chromatography and chemometric analysis. Food Chemistry, 136, 961-968.

Mayer, B.X. and Lorbeer, E. (1997). Triacylglycerol mixture for testing capillary columns for high-temperature gas chromatography. Journal of Chromatography A, 758, 235-242.

Morrison, D.J., Cooper, K. and Pretson, T. (2010). Reconstructing bulk isotope ratios from compound-specific isotope ratios. Rapid Communications in Mass Spectrometry, 24, 1799-1804.

Paolini, M., Bontempo, L. and Camin, F. (2017). Compoundspecific $\square^{13} \mathrm{C}$ and $\square^{2} \mathrm{H}$ analysis of olive oil fatty acids. Talanta, 174, 38-43.

Qi, H., Coplen, T.B., Geilmann, H., Brand, W.A. and Böhlke, J.K. (2003). Two new organic reference materials for $\delta^{13} \mathrm{C}$ and $\delta^{15} \mathrm{~N}$ measurements and a new value for the $\delta^{13} \mathrm{C}$ of NBS 22 oil. Rapid Communications in Mass Spectrometry, 17, 2483-2487.

Pereira, A.S., Siqueira, D.S., Elias, V.O., Simoneit, B.R.T., Cabral, J.A. and Aquino Neto, F.R. (2002). Three series of high molecular weight alkanoates found in Amazonian plants. Phytochemistry, 61, 711-719.

Pereira, A.S., Bicalho, B. and Aquino Neto, F.R. (2003). Comparison of propolis from Apis mellifera and Tetragonisca angustula. Apidologie, 34, 291-298.

Ruiz-Samblás, C., González-Casado, A., Cuadros-Rodríguez, L. and Rodríguez García, F.P. (2010). Application of selected ion monitoring to the analysis of triacylglycerols in olive oil by high temperature-gas chromatography/mass spectrometry. Talanta, 82, 255-260. 
Ruiz-Samblás, C., Cuadros-Rodríguez, L., González-Casado, A. and Rodríguez García, F.P. (2012). A straightforward quantificataion of triacylglycerols (and fatty acids) in monovarietal extra virgin olive oils by high-temperature GC. Analytical Methods, 4, 753-758.

Ruiz-Samblás, C., González-Casado, A. and Cuadros-Rodríguez, L. (2015). Triacylglycerols determination by high-temperature gas chromatography in the analysis of vegetable oils and foods: A Review of the Past 10 Years. Critical Reviews in Food Science and Nutrition, 55, 1618-1631.

Sutton, P.A. and Rowland, S.J. (2012). High temperature gas chromatography-time-of-flight mass spectrometry (HTGC-ToF-
MS) for high-boiling compounds. Journal of Chromatography A, 1243, 69-80.

Tena, N, Wang, S.C., Aparicio-Ruiz, R., García-González, D.L. and Aparicio, R. (2015). In-depth assessment of analytical methods for olive oil purity, safety, and quality characterization. Journal of Agricultural and Food Chemistry, 63, 4509-4526.

Wei, G-L. and Zeng, E.Y. (2011). Gas chromatography-mass spectrometry and high-performance liquid chromatographytandem mass spectrometry in quantifying fatty acids. Trends in Analytical Chemistry, 30, 1429-1436. 